\title{
New Terms for the Compact Form of Electroweak Chiral Lagrangian
}

\author{
Hong-Hao Zhang, Wen-Bin Yan, and J. K. Parry \\ Center for High Energy Physics \& Department of Physics, Tsinghua University, \\ Beijing 100084, China \\ E-mail: zhanghonghao@tsinghua.org.cn, wenbin.yan@gmail.com, \\ jkparry@tsinghua.edu.cn

\section{Xue-Song Li} \\ Science College, Hunan Agricultural University, Changsha 410128, China \\ E-mail: 1ixuesong@tsinghua.org.cn
}

\begin{abstract}
The compact form of the electroweak chiral Lagrangian is a reformulation of its original form and is expressed in terms of chiral rotated electroweak gauge fields, which is crucial for relating the information of underlying theories to the coefficients of the lowenergy effective Lagrangian. However the compact form obtained in previous works is not complete. In this letter we add several new chiral invariant terms to it and discuss the contributions of these terms to the original electroweak chiral Lagrangian.
\end{abstract}

Keywords: Electroweak Chiral Lagrangian, Beyond Standard Mode]. 
So far the postulated Higgs particle of the standard model has not been observed in experiments. We do not know what the electroweak symmetry breaking mechanism in nature is. The electroweak chiral Lagrangian is a general low-energy description for electroweak symmetry breaking patterns [1], 2], especially for those strong dynamical electroweak symmetry breaking mechanisms [3]. All the coefficients of the electroweak chiral Lagrangian, in principle, can be fixed by experiments $14,5,6,0,8,9,10,11,12,13$. Due to the nonperturbative property of the possible strong dynamics, it is difficult to relate the measured coefficients of the electroweak chiral Lagrangian to underlying theories. Recently, the series of work of Ref. 114, 15, 16, 17, 18, 19, 20, 21] successfully produced the predictions for the coefficients of the chiral Lagrangian from the underlying theory of QCD, which lights up the hope of building up the relationship between the coefficients of the electroweak chiral Lagrangian and underlying strong dynamical models. As seen in Ref. [14], a crucial first step of the derivation of the chiral Lagrangian from QCD is to reformulate the original chiral Lagrangian in terms of chiral rotated external source fields. For the case of the electroweak chiral Lagrangian, we need to similarly reformulate it in terms of chiral rotated electroweak gauge fields in order to deduce the information of underlying theories. An attempt of this line of thought is the work of Ref. [22], which tries to give a so-called compact form of the electroweak chiral Lagrangian in terms of chiral rotated electroweak gauge fields. However, there are still several relevant terms not included in the reformulation of Ref. [22]. In this letter, we shall present 3 new terms for this reformulation and give their contributions to the original electroweak chiral Lagrangian.

We refer the interested reader to Ref. [22] for all the details on the compact form of the electroweak chiral Lagrangian and the relations between the compact form and the original form. Besides the inner-product terms $c_{2} g_{2}^{4} V_{\mu}^{a^{\prime}} V_{\nu}^{a^{\prime}} V^{b^{\prime} \mu} V^{b^{\prime} \nu}, c_{8} g_{2}^{2}\left(d_{\mu} V_{\nu}^{a^{\prime}}-d_{\nu} V_{\mu}^{a^{\prime}}\right) V^{a^{\prime} \mu} A^{\nu}$, and $c_{9} g_{2}^{2}\left(d^{\mu} V_{\mu}^{a^{\prime}}\right) V_{\nu}^{a^{\prime}} A^{\nu}$ already included in the compact form of Ref. [22], there should be 3 new cross-product terms which are given by,

$$
\Delta \mathcal{L}=c_{2}^{\prime} g_{2}^{4}\left(\epsilon^{a^{\prime} b^{\prime}} V_{\mu}^{a^{\prime}} V_{\nu}^{b^{\prime}}\right)^{2}+c_{8}^{\prime} g_{2}^{2} \epsilon^{a^{\prime} b^{\prime}}\left(d_{\mu} V_{\nu}^{a^{\prime}}-d_{\nu} V_{\mu}^{a^{\prime}}\right) V^{b^{\prime} \mu} A^{\nu}+c_{9}^{\prime} g_{2}^{2} \epsilon^{a^{\prime} b^{\prime}}\left(d^{\mu} V_{\mu}^{a^{\prime}}\right) V_{\nu}^{b^{\prime}} A^{\nu}
$$

where $a^{\prime}, b^{\prime}$ run from 1 to 2 , and $\epsilon^{12}=-\epsilon^{21}=1$. Here and henceforth $V^{a^{\prime} \mu}$ and $A^{\mu}$ are short for the chiral rotated gauge fields $V_{\xi}^{a^{\prime} \mu}$ and $A_{\xi}^{\mu}$ in Ref. 22] respectively. There are also 3 cross-product terms corresponding to the $c_{15,17,25}$-terms in that paper, but they vanish. Let us consider Eq. (11) term by term. From the definitions of Ref. [22], it is straightforward to obtain the relation between the first term of Eq. (11) and the ordinary terms of the electroweak chiral Lagrangian as follows,

$$
\begin{aligned}
c_{2}^{\prime} g_{2}^{4}\left(\epsilon^{a^{\prime} b^{\prime}} V_{\mu}^{a^{\prime}} V_{\nu}^{b^{\prime}}\right)^{2}= & -4 c_{2}^{\prime}\left[\left[\operatorname{tr}\left(X_{\mu} X_{\nu}\right)\right]^{2}-\left[\operatorname{tr}\left(X_{\mu} X^{\mu}\right)\right]^{2}-\operatorname{tr}\left(X_{\mu} X_{\nu}\right) \operatorname{tr}\left(\tau^{3} X^{\mu}\right) \operatorname{tr}\left(\tau^{3} X^{\nu}\right)\right. \\
& \left.+\operatorname{tr}\left(X_{\mu} X^{\mu}\right)\left[\operatorname{tr}\left(\tau^{3} X_{\nu}\right)\right]^{2}\right]
\end{aligned}
$$

where $X_{\mu} \equiv U^{\dagger}\left(D_{\mu} U\right)$. And the last two terms of Eq. (西) are respectively given by,

$$
c_{8}^{\prime} g_{2}^{2} \epsilon^{a^{\prime} b^{\prime}}\left(d_{\mu} V_{\nu}^{a^{\prime}}-d_{\nu} V_{\mu}^{a^{\prime}}\right) V^{b^{\prime} \mu} A^{\nu}
$$




$$
\begin{aligned}
= & c_{8}^{\prime}\left[-\frac{1}{2} \operatorname{tr}\left(\tau^{3} X^{\nu}\right)\left[\operatorname{tr}\left(\tau^{3} X^{\mu}\right) \operatorname{tr}\left(X_{\mu} X_{\nu}\right)-\operatorname{tr}\left(\tau^{3} X_{\nu}\right) \operatorname{tr}\left(X_{\mu} X^{\mu}\right)\right]+i \operatorname{tr}\left(\bar{W}_{\mu \nu} X^{\mu} X^{\nu}\right)\right. \\
& \left.-\frac{i}{2} \operatorname{tr}\left(\tau^{3} \bar{W}_{\mu \nu}\right) \operatorname{tr}\left(\tau^{3} X^{\mu} X^{\nu}\right)\right]
\end{aligned}
$$

with $\bar{W}_{\mu \nu} \equiv U^{\dagger} g_{2} \frac{\tau^{a}}{2} W_{\mu \nu}^{a} U$, and

$$
\begin{aligned}
c_{9}^{\prime} g_{2}^{2} \epsilon^{a^{\prime} b^{\prime}}\left(d^{\mu} V_{\mu}^{a^{\prime}}\right) V_{\nu}^{b^{\prime}} A^{\nu}= & \frac{1}{2}\left(1-4 \beta_{1}\right) c_{9}^{\prime}\left[\operatorname{tr}\left(\tau^{3} X^{\mu}\right) \operatorname{tr}\left(\tau^{3} X^{\nu}\right) \operatorname{tr}\left(X_{\mu} X_{\nu}\right)\right. \\
& \left.-\left[\operatorname{tr}\left(\tau^{3} X_{\mu}\right) \operatorname{tr}\left(\tau^{3} X_{\nu}\right)\right]^{2}\right] .
\end{aligned}
$$

From Eqs. (2), (3) and (4), we obtain the contributions of these 3 new terms to the original electroweak chiral Lagrangian as follows,

$$
\begin{aligned}
& \Delta \alpha_{3}=\frac{c_{8}^{\prime}}{2}, \quad \Delta \alpha_{4}=-4 c_{2}^{\prime}, \quad \Delta \alpha_{5}=4 c_{2}^{\prime}, \\
& \Delta \alpha_{6}=4 c_{2}^{\prime}-\frac{c_{8}^{\prime}}{2}+\frac{1}{2}\left(1-4 \beta_{1}\right) c_{9}^{\prime}, \quad \Delta \alpha_{7}=-4 c_{2}^{\prime}+\frac{c_{8}^{\prime}}{2}, \\
& \Delta \alpha_{9}=-\frac{c_{8}^{\prime}}{2}, \quad \Delta \alpha_{10}=\left(1-4 \beta_{1}\right) c_{9}^{\prime} .
\end{aligned}
$$

The coefficients $c_{2,8,9}^{\prime}$ and $c_{i}(i=1,2, \ldots, 25)$ in this compact form of the electroweak chiral Lagrangian are determined by the underlying ultraviolet theories. For example, if we take the one-doublet technicolor model as the underlying theory, it can be shown that these 3 new coefficients $c_{2,8,9}^{\prime}$ are all non-vanishing, and full details will be presented in forthcoming publications [23].

In summary, we have provided 3 new terms to the compact form of the electroweak chiral Lagrangian introduced in Ref. [22]. These additional terms were not considered in the previous work. In this letter we have related these new terms to the original electroweak chiral Lagrangian, which will be crucial in forthcoming studies of strong dynamical models.

\section{Acknowledgments}

We are indebted to Qing Wang for all our knowledge about the chiral Lagrangian and his helps and supports for this work. This work is supported in part by the National Natural Science Foundation of China.

\section{References}

[1] T. Appelquist and C. W. Bernard, Phys. Rev. D 22, 200 (1980). T. Appelquist and G. H. Wu, Phys. Rev. D 48, 3235 (1993) [arXiv:hep-ph/9304240].

[2] A. C. Longhitano, Phys. Rev. D 22, 1166 (1980); Nucl. Phys. B 188, 118 (1981).

[3] C. T. Hill and E. H. Simmons, Phys. Rept. 381, 235 (2003) [Erratum-ibid. 390, 553 (2004)] [arXiv:hep-ph/0203079]. 
[4] J. F. Donoghue, C. Ramirez and G. Valencia, Phys. Rev. D 39, 1947 (1989); J. F. Donoghue and C. Ramirez, Phys. Lett. B 234, 361 (1990). A. Dobado and M. T. Urdiales, Z. Phys. C 71, 659 (1996) [arXiv:hep-ph/9502255].

[5] H. J. He, Y. P. Kuang and C. P. Yuan, Phys. Lett. B 382, 149 (1996) [arXiv:hep-ph/9604309]; Phys. Rev. D 55, 3038 (1997) [arXiv:hep-ph/9611316]; "Global analysis for probing electroweak symmetry breaking mechanism at high energy colliders," arXiv:hep-ph/9704276.

[6] H. J. He, "Quartic gauge boson couplings," arXiv:hep-ph/9804210, Invited overview talk published in the Proceedings of Workshop on Physics at the First Muon Collider, pp. 685-700, Fermilab, Batavia, USA, Nov. 6-9, 1997; T. Han, H. J. He and C. P. Yuan, Phys. Lett. B 422, 294 (1998) [arXiv:hep-ph/9711429].

[7] S. Alam, S. Dawson and R. Szalapski, Phys. Rev. D 57, 1577 (1998) [arXiv:hep-ph/9706542].

[8] H. J. He, Y. P. Kuang, C. P. Yuan and B. Zhang, Phys. Lett. B 554, 64 (2003) [arXiv:hep-ph/0211229].

[9] B. Zhang, Y. P. Kuang, H. J. He and C. P. Yuan, Phys. Rev. D 67, 114024 (2003) [arXiv:hep-ph/0303048].

[10] H. J. He, Y. P. Kuang, C. P. Yuan and B. Zhang, arXiv:hep-ph/0401209, Published in the Proceedings of the 3rd Les Houches Workshop: Physics at TeV Colliders, Les Houches, France, May 26-Jun 6, 2003.

[11] R. S. Chivukula, E. H. Simmons, H. J. He, M. Kurachi and M. Tanabashi, Phys. Rev. D 72, 075012 (2005) [arXiv:hep-ph/0508147].

[12] T. Han, Y. P. Kuang and B. Zhang, Phys. Rev. D 73, 055010 (2006) [arXiv:hep-ph/0512193].

[13] E. Boos, H. J. He, W. Kilian, A. Pukhov, C. P. Yuan and P. M. Zerwas, Phys. Rev. D 57, 1553 (1998) [arXiv:hep-ph/9708310]; Phys. Rev. D 61, 077901 (2000) [arXiv:hep-ph/9908409]. M. Beyer, W. Kilian, P. Krstonosic, K. Monig, J. Reuter, E. Schmidt and H. Schroder, Eur. Phys. J. C 48, 353 (2006) [arXiv:hep-ph/0604048].

[14] Q. Wang, Y. P. Kuang, M. Xiao and X. L. Wang, Phys. Rev. D 61, 054011 (2000) [arXiv:hep-ph/9903201].

[15] Q. Wang, Y. P. Kuang, X. L. Wang and M. Xiao, arXiv:hep-ph/9910289.

[16] X. L. Wang, Z. M. Wang and Q. Wang, Commun. Theor. Phys. 34, 683 (2000).

[17] X. L. Wang and Q. Wang, Commun. Theor. Phys. 34, 519 (2000).

[18] H. Yang, Q. Wang, Y. P. Kuang and Q. Lu, Phys. Rev. D 66, 014019 (2002) [arXiv:hep-ph/0203040].

[19] Q. Wang, Y. P. Kuang, H. Yang and Q. Lu, J. Phys. G 28, L55 (2002) [arXiv:hep-ph/0209201].

[20] Q. Wang, Int. J. Mod. Phys. A 20, 1627 (2005).

[21] Y. L. Ma and Q. Wang, Phys. Lett. B 560, 188 (2003) [arXiv:hep-ph/0302143].

[22] Z. M. Wang and Q. Wang, Commun. Theor. Phys. 36, 417 (2001).

[23] H. H. Zhang, S. Z. Jiang and Q. Wang, "Dynamical Computation on Coefficients of Electroweak Chiral Lagrangian from One-doublet and Topcolor-assisted Technicolor Models," arXiv:0705.0115 [hep-ph]. 